\section{Interferon's HCV interference}

\section{By Brian Moy, Staff Writer}

Standard therapy for chronic HCV infection-PEGylated interferon$\alpha$ and the antiviral ribavirin-only achieves sustained viral clearance in about $55 \%$ of infected patients. ${ }^{1}$ A paper in the Proceedings of the National Academy of Sciences suggests that activation of the endogenous interferon system prior to therapy could be responsible for that low efficacy.

The findings have both therapeutic and diagnostic implications. On the treatment side, the research may help clinicians develop a strategy to reverse preactivation of interferon (IFN) and restore responsiveness to IFN therapeutics. On the diagnostic front, the work could lead to tests that identify potential responders to standard therapy.

The study, conducted by researchers at the University of Basel in collaboration with the Friedrich Miescher Institute for Biomedical Research, examined liver biopsies from 16 patients with chronic HCV before and after treatment with standard therapy. Analysis of IFN-induced signaling and IFN-stimulated genes (ISGs) showed that ISG expression was upregulated following standard therapy
"An important question to answer is whether the failure of liver cells to rid themselves of viral infection in nonresponders is a result of the virus itself or whether it is due to an intrinsic defect in the interferon signaling pathway."

- John Alam, Vertex Pharmaceuticals Inc.
Another potential explanation is that the virus does not infect the majority of hepatocytes and that the induction of ISGs "could occur predominantly in noninfected hepatocytes," the authors wrote. In infected liver cells, the virus would retain its ability to inhibit IFN- $\alpha$ signaling ${ }^{3}$ and would be able to shrug off any IFN secreted by neighboring, noninfected cells.

Robert Ralston, senior director of virology at Schering-Plough Corp., said the PNAS study "is an important advance in our understanding of the action of interferon- $\alpha$ in chronic HCV patients and, in particular, why some patients fail to respond to interferon therapy."

\section{Behind the failure}

A key question is what actually causes preactivated IFN signaling in nonresponsive HCV patients.

"If we can find the molecular mechanisms responsible for preactivation of endogenous interferon in the liver, we can potentially develop a strategy to reverse this preactivation and turn nonresponders into responders," said Markus Heim, principal investigator and corresponding author of the PNAS paper. Heim is a professor of medicine at the University of Basel.

The authors are now investigating the causes of such preactivation.

To solve the mystery, John Alam, EVP of medicines development and CMO at Vertex Pharmaceuticals Inc., said "an important question to answer is whether the failure of liver cells to rid themselves of viral infection in nonresponders is a result of the virus itself or whether it is due to an intrinsic defect in the interferon signaling pathway."

Previous studies suggest the ability of in patients who responded to treatment, whereas expression in nonresponders was initially high before treatment and did not change afterward. ${ }^{2}$

Moreover, the study identified a subset of ISGs whose expression was highly predictive of treatment outcome. Several ISGs had significantly higher pretreatment expression in patients with the difficult-to-treat $\mathrm{HCV}$ genotypes 1 and 4 than in those with genotypes 2 and 3.

The researchers had a few hypotheses about why high levels of endogenous IFN are linked to treatment nonresponders.

"In patients without a preactivated IFN system, the injection of exogenous IFN- $\alpha$ during treatment should induce an antiviral state very rapidly in most liver cells, and HCV would not have 'enough' time to escape from the IFN-induced defense," according to the authors.

In contrast, in patients with preactivated IFN, the virus might have "enough time to adapt to and evade the intracellular antiviral defense system, making it also resistant to the subsequent IFN therapy," the authors said.
$\mathrm{HCV}$ to interfere with the IFN pathway at many different levels is a likely mechanism behind the virus's ability to establish chronic infection. ${ }^{4}$

In the PNAS paper, both pre- and post-treatment expression of ISGs in HCV patients responding to standard therapy did not exceed the expression observed in nonresponders. According to the authors, this finding suggests there could be defects downstream of ISG expression in patients with a preactivated IFN system.

Alam, however, thinks the problem "is not an intrinsic defect within the interferon signaling pathway, but rather that it is virally mediated. Because viruses have mechanisms to keep the interferon system from clearing them, it seems likely that cells infected with $\mathrm{HCV}$ are not able to activate all of the downstream genes and proteins of the interferon pathway that are required to eliminate infection."

Lawrence Blatt, president and CEO of Alios BioPharma Inc., agreed that there is probably not an inherent defect in downstream IFN signaling. "There is no reason to suspect that there is a selection bias for infection with different HCV genotypes in patients with 
different interferon-responsiveness phenotypes," he said. "Thus, the resistance to interferon must be mediated by viral factors inherent in the specific HCV infection."

Alios is developing Glycoferon, a long-acting type I IFN, which is in preclinical testing to treat $\mathrm{HCV}$. Blatt said that "by giving a more potent interferon, such as Glycoferon, we can overcome the HCVmediated blockade of interferon response, which can potentially lead to sustained virological responses in patients who have previously failed standard therapy."

In vitro, the company says, Glycoferon has shown 500-fold greater biological potency than PEGylated IFN- $\alpha$.

\section{Therapeutic implications}

If $\mathrm{HCV}$ itself is responsible for preactivating IFN and blocking the host's IFN system from clearing the virus, Vertex's Alam thinks there are clear implications for treating the disease with small molecules.

"From a therapeutic standpoint, if you are able to shut off viral replication and block the production of viral protein, you would restore the cells' ability to rid themselves of HCV infection and convert nonresponders and null responders into responders," he said.

Vertex is developing telaprevir, a small molecule HCV NS3 protease inhibitor in Phase III testing to treat HCV. The company also has two second-generation HCV protease inhibitors: VX-500, in Phase Ia testing, and VX-813, in preclinical development.

Last month, Vertex announced results from a Phase II trial of telaprevir that included patients with HCV genotype 1 infection who were null responders, nonresponders or who had relapsed following standard therapy in previous Phase II trials. In those patients, telaprevir plus standard therapy with Pegasys peginterferon alfa-2a and Copegus ribavirin reduced HCV RNA at week 4 to $<25 \mathrm{IU} / \mathrm{ml}$ in 18 of 24 null responders, 18 of 19 partial responders and 5 of 6 patients who relapsed. Roche markets Pegasys and Copegus.

"Because telaprevir specifically targets the virus, the only way it would be able to reduce HCV RNA levels in patients previously nonresponsive to standard therapy as consistently as we have seen is by reversing a block that is produced by the virus," said Alam.

The finding that patients with a preactivated endogenous IFN system do not respond to IFN- $\alpha$ therapy also could have important implications for new classes of HCV antivirals. Schering-Plough's Ralston said that "as targeted small molecule antivirals such as HCV protease inhibitors are added to peginterferon-containing regimens, it will be important to know whether or not a patient can respond to interferon to ensure that all drugs in the regimen are active."

He added that "clinical monitoring of viral load decline in response to initial peginterferon therapy before addition of the targeted small molecule to the regimen provides an alternative method for assessing a patient's ability to respond to interferon." Schering-Plough is using this approach in clinical studies of boceprevir in combination with peginterferon and ribavirin. The HCV NS3 protease inhibitor is in Phase II testing.

Schering-Plough and Enzon Pharmaceuticals Inc. market PEGIntron, a PEGylated recombinant interferon alfa-2b, and Rebetol ribavirin for $\mathrm{HCV}$ infection.

\section{REFERENCES}

1. Hadziyannis, S.J. et al. Ann. Intern. Med. 140, 346-355 (2004)

2. Sarasin-Filipowicz, M. et al. Proc. Natl. Acad. Sci. USA; published online May 5, 2008; doi:10.1073/pnas.0707882105

Contact: Markus H. Heim, University Hospital Basel, Basel, Switzerland e-mail: markus.heim@unibas.ch

3. Christen, V. et al. Hepatology 46, 558-565 (2007)

4. Gale, M. \& Foy, E. Nature 436, 939-945 (2005)

COMPANIES AND INSTITUTIONS MENTIONED

Alios BioPharma Inc., South San Francisco, Calif.

Enzon Pharmaceuticals Inc. (NASDAQ:ENZN), Bridgewater, N.J. Friedrich Miescher Institute for Biomedical Research, Basel, Switzerland Roche (SWX:ROG), Basel, Switzerland

Schering-Plough Corp. (NYSE:SGP), Kenilworth, N.J.

University of Basel, Basel, Switzerland

Vertex Pharmaceuticals Inc. (NASDAQ:VRTX), Cambridge, Mass. 